\title{
Insulin X10 revisited: a super-mitogenic insulin analogue
}

\author{
B. F. Hansen • P. Kurtzhals • A. B. Jensen • \\ A. Dejgaard $\cdot$ D. Russell-Jones
}

Received: 22 December 2010 / Accepted: 6 April 2011 /Published online: 3 June 2011

(C) Springer-Verlag 2011

\begin{abstract}
The molecular safety of insulin analogues has received a great deal of attention over the last year. In particular, attention has been directed to the mitogenic properties of insulin analogues as compared with human insulin. Understanding the mechanisms implicated in mediating mitogenic effects of insulin is therefore of particular interest. In this review we detail the story of the rapid-acting insulin analogue known as X10, which was the first insulin analogue in clinical development, but ended up being discontinued at an early clinical development stage following findings of mammary tumours in female Sprague-Dawley rats. The molecular characteristics of insulin X10, along with its interaction at both the IGF-1 receptor and the insulin receptor, have provided us with important insights into mechanisms implicated in metabolic and mitogenic signalling of insulin analogues.
\end{abstract}

Keywords Dissociation rate $\cdot$ IGF-1 receptor - Insulin analogue Insulin receptor - Insulin X10 • Mitogenicity · Receptor affinity $\cdot$ Review

B. F. Hansen $\cdot$ P. Kurtzhals $\cdot$ A. Dejgaard

Novo Nordisk A/S,

Bagsvaerd, Denmark

A. B. Jensen

Bryggen Medical Center,

Hammerfest, Norway

D. Russell-Jones $(\triangle)$

The Cedar Centre, Royal Surrey County Hospital,

Egerton Road,

Guildford, Surrey GU2 7XX, UK

e-mail: davidrussell-jones@nhs.net

\author{
Abbreviations \\ IGF-1R IGF-1 receptor \\ IR Insulin receptor \\ PI3K Phosphatidylinositol 3-kinase \\ ERK Extracellular signal-regulated kinase \\ MCF Michigan Cancer Foundation \\ HMEC Human mammary epithelial cells
}

\section{Introduction}

Type 2 diabetes is associated with an increased risk of a range of cancers $[1,2]$. In addition, comorbidities and lifestyle factors that are commonly associated with type 2 diabetes, such as obesity and physical inactivity, are associated with an increased risk [3]. It is known that different glucose-lowering treatments can modify the risk of developing cancer in different ways, with metformin apparently providing a protective effect and insulin secretagogues and insulin potentially stimulating tumour growth $[4,5]$. The publication of four epidemiological studies in June 2009 highlighted a potential link between insulin glargine (A21Gly,B31Arg,B32Arg human insulin) and an increased incidence of cancer, stirring up an ongoing debate about the long-term clinical safety of insulin analogues [6-9].

There is one molecule that is particularly interesting in relation to this recent development. Insulin X10 is a human insulin analogue in which the histidine-B10 residue has been replaced with an aspartic acid residue, resulting in a rapid-acting insulin analogue $[10,11]$. After the discovery that, in 52 week toxicity studies, insulin X10 was associated with an increased occurrence of mammary tumours in female Sprague-Dawley rats, development of this analogue was discontinued [12]. Here we report on the 
characteristics of insulin X10 and the knowledge gained from subsequent investigations into the mechanism of its increased mitogenic action.

\section{The development of insulin X10}

The rationale for developing a rapid-acting insulin analogue is to overcome the pharmacokinetic limitations of subcutaneously injected human insulin for postprandial glycaemic control. Because human insulin naturally self-associates into hexamers at the concentrations found in the subcutaneous injection depot, it displays a somewhat protracted absorption rate into the bloodstream, limiting its usefulness for postprandial control. However, this self-association can be disrupted, and an insulin molecule that does not selfassociate into hexamers, or does so more weakly, is absorbed from the injection depot into the bloodstream much more quickly. With the advent of recombinant DNA technology, single amino acid substitutions could be targeted to regions involved in hexamer formation that also lie on the periphery of the insulin receptor (IR) binding regions [10]. The histidine residue at the B10 position was known to be involved in self-association through the coordination of zinc ions by the imidazole side chains [13]. One of the analogues described by Brange and colleagues [10] replaced this histidine residue with the negatively charged aspartic acid, creating the insulin analogue known as insulin X10. The ability of insulin $\mathrm{X} 10$ to self-associate into hexamers was successfully disrupted, even at concentrations of $100 \mathrm{U} / \mathrm{ml}$. After subcutaneous injection into pigs, the time taken for $50 \%$ of the dose to be absorbed from the injection depot was found to be approximately half that of human insulin. In addition to the increased rate of absorption, insulin X10 also displayed a higher maximum concentration and a more potent biological action than natural insulin.

The rapid absorption rate from the subcutaneous depot demonstrated by Brange and colleagues made insulin X10 an interesting candidate for further research with a view to developing a rapid-acting insulin analogue. Subsequent studies confirmed that the rapid rate of absorption from the subcutaneous injection depot seen in pigs also occurred both in healthy volunteers and in patients with type 1 diabetes [14, 15]. In a long-term double-blind, randomised crossover study with treatment periods of 2 months involving 21 male patients with type 1 diabetes, insulin X10 was shown to have a more physiological plasma profile than soluble human insulin [16]. With insulin X10, there were significantly lower postprandial blood glucose levels in the morning, similar glucose excursions at lunch and dinner, and slightly higher levels during the night, resulting in similar $\mathrm{HbA}_{1 \mathrm{c}}$ levels achieved with insulin $\mathrm{X} 10$ and human insulin at the end of the 2 month treatment periods. There were no differences in reported side effects, including the frequency of mild hypoglycaemic events, between the two insulins.

Thus, all the evidence suggested that insulin X10 was a promising new rapid-acting insulin analogue that facilitated a reduction in $\mathrm{HbA}_{1 \mathrm{c}}$ that was very similar to that shown with human insulin, whilst at the same time providing a more physiological postprandial plasma profile. But then came the discovery that halted any future clinical development of insulin X10. A 52 week study of supraphysiological exposure in rats revealed a dose-dependent increase in the occurrence of mammary tumours in female Sprague-Dawley rats [12]. In the group exposed to the highest dose (200 $\mathrm{U} \mathrm{kg}^{-1}$ day $\left.^{-1}\right)$, $44 \%$ of female rats developed benign tumours and $23 \%$ developed malignant tumours after 1 year (Fig. 1). A 10 year follow-up of all patients (all male) exposed to more than a single dose of insulin X10 in the study by Nielsen and colleagues [16] revealed no increased incidence of malignancies. Nevertheless, the rat data prompted a series of investigations into the cause of this increased risk, focused primarily on mitogenic potency, as well as on the IR and IGF-1 receptor (IGF-1R) binding characteristics of insulin X10.

\section{Understanding insulin X10}

One of the first molecular characteristics that was observed to differ between insulin X10 and human insulin was increased binding to IR itself $[11,17]$. In the majority of studies the binding affinity of insulin X10 to IR has been found to be increased $200-400 \%$ relative to that of human insulin. It was also noted by these early studies that the binding affinity of insulin X10 to IGF-1R was increased compared with that of human insulin $[17,18]$ but with a fairly low affinity compared with IGF-1 itself, an effect that has subsequently been confirmed by a number of authors [19-22]. In addition to the increased receptor affinities,

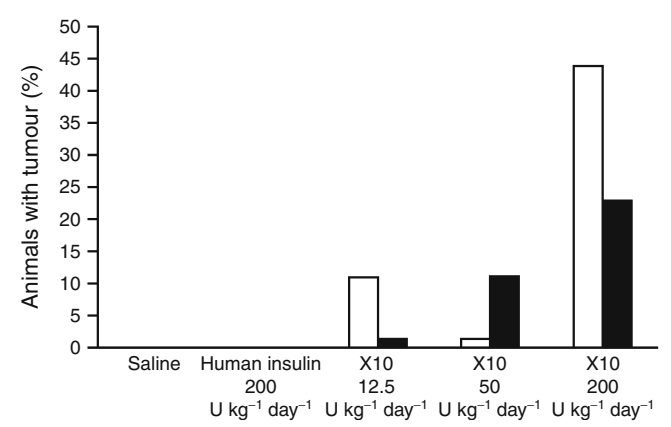

Fig. 1 Tumour incidence in Sprague-Dawley rats after exposure to insulin X10. Based on Drejer et al. [12] 


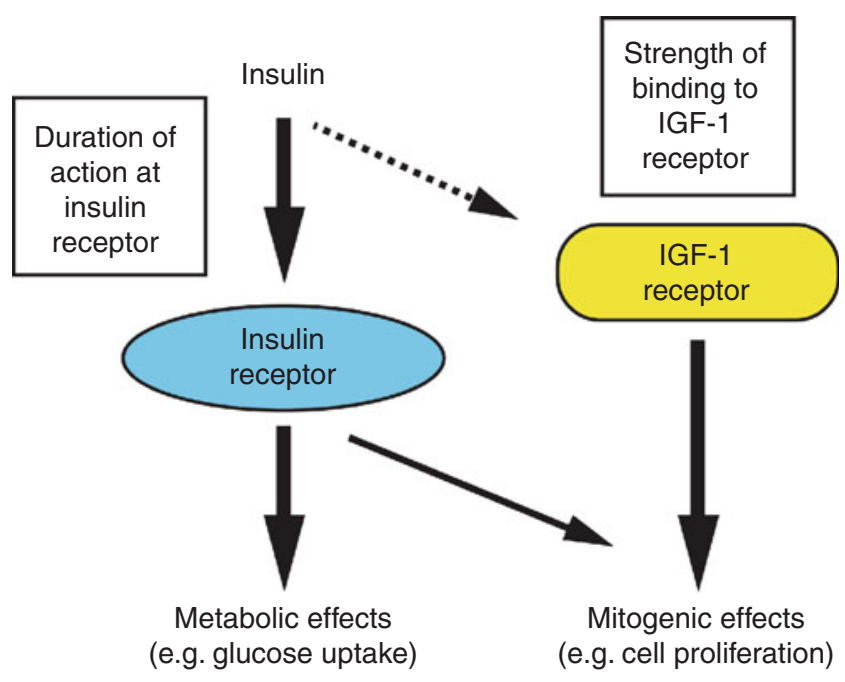

Fig. 2 Molecular characteristics potentially influencing the balance of metabolic and mitogenic actions of insulin-like molecules

insulin X10 was shown to have an increased in vitro ability to stimulate cell growth and DNA synthesis, and Bornfeldt and colleagues speculated that this was due to a substitution in the insulin X10 molecule that made this analogue more chemically similar to the IGF-1 molecule [18]. Compared with human insulin, insulin X10 had an identical 'on-rate' but a much slower 'off-rate' from the IR so that, upon binding, insulin X10 remained bound for much longer than human insulin [17].

These early observations led to a series of experiments with insulin X10 and other analogues which showed that at least two molecular characteristics can be responsible for the increased mitogenic potency of insulin analogues (Fig. 2).

A correlation between relative IGF-1R affinity and mitogenic potency was described by Slieker and colleagues [20] and Kurtzhals and co-workers [19] and later by Kohn and colleagues [23] (Fig. 3). Intuitively this mechanism seems

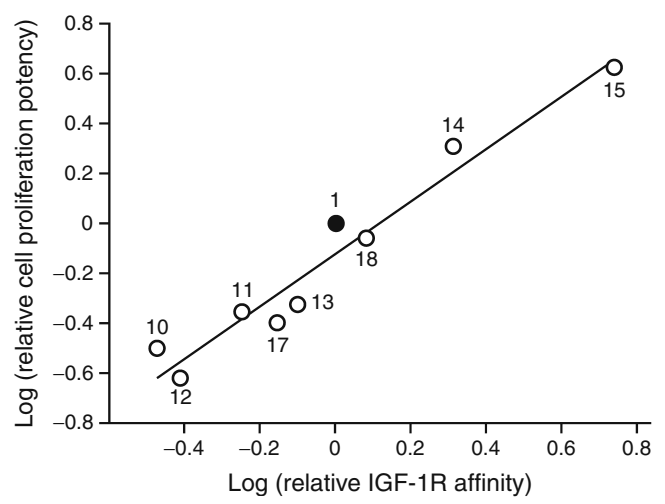

Fig. 3 Relation between increased IGF-1R affinity of insulin analogue molecules and their mitogenic potential. Reprinted from Kohn et al. [23] with permission from Elsevier. 1 Human insulin. $10 \mathrm{~A} 0: \mathrm{R}, \mathrm{A}(-1): \mathrm{R}$, A21:G. 11 B0:R, B(-1):R, A21:G. 12 A0:R, B0:R, A21:G. 13 A0:R, B31:R, A21:G. 14 B0:R, B31:R, A21:G. 15 B31:R, B32:R, A21:G. 17 A0:R, A(-1):R, B31:R, A21:G. 18 A0:R, B31:R, B32:R, A21:G plausible - increased affinity for IGF-1R results in an increased ability to promote cell growth. In addition to the mechanism mediated via IGF-1R, it was shown that the mitogenic potency of insulin analogues was increased in excess of the metabolic potency when the dissociation rate from IR was decreased. Thus, the decreased dissociation rate observed with insulin X10 [17] was extended to a range of insulin analogues, and a hyperbolic relationship between the dissociation rate constant and the ratio between mitogenic and metabolic potency (Fig. 4) was demonstrated [24].

The ability of insulin X10 to promote tumour growth could be explained by either, or both, of these molecular characteristics, and it would therefore be rational to avoid either mechanism in the development of new insulin analogues even if it is not known for certain if any of these in vitro observations were responsible for the findings in vivo in rats.

If we try to determine the relative importance of these two molecular characteristics, the finding that knockdown of IGF-1R by small interfering RNAs largely abolishes the mitogenic effect of IGF-1 and insulin analogues indicates that increased IGF-1R affinity might be more important $[25,26]$. Also, in the study of Kurtzhals and colleagues on

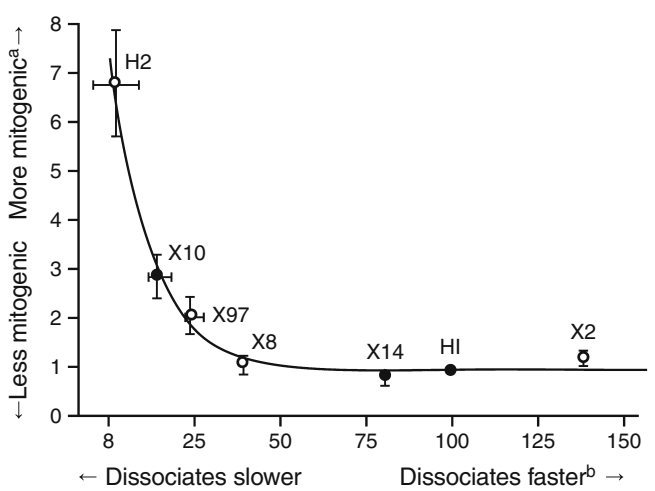

Fig. 4 Relation between dissociation rate from the insulin receptor of insulin analogue molecules and their mitogenic potential. ${ }^{\text {a Ratio of }}$ mitogenic:metabolic activity. ${ }^{\mathrm{b}}$ Dissociation rate constant $\left(K_{\mathrm{d}}\right)$. Dissociation was analysed by incubating Chinese hamster ovary cells overexpressing the human insulin receptor (CHO-hIR cells) with ${ }^{125}$ I-labelled insulin or analogue for $3 \mathrm{~h}$ at $4^{\circ} \mathrm{C}$. Cells were then washed quickly twice and dissociation determined after the addition of $0.1 \mu \mathrm{mol} / 1$ unlabelled insulin or analogue. Cell-associated radioactivity was measured as a function of time and residual binding expressed as a percentage of initial binding. The dissociation rate constants $\left(K_{\mathrm{d}}\right)$ were calculated using the LIGAND program (National Institutes of Health, Bethesda, MD, USA). The mitogenic/metabolic ratio was calculated as the proportion between relative mitogenic potency and relative metabolic potency. The mitogenic potency was measured as thymidine incorporation into CHO-hIR cells and the metabolic potency as glucose transport in primary adipocytes. Insulin: $X 2$, [B-Asp9,B-Glu27]insulin; $H I$, human insulin; X14, [B-Asp28] insulin; X8, [A-His8]insulin; X10, [B-Asp10]insulin; X97, [B-Glu10,B-des-Thr30]insulin; H2, [A-His8,B-His4,B-Glu10,B-His27] insulin. This research was originally published in Biochem $J$. Hansen BF et al. [24] Sustained signalling from the insulin receptor after stimulation with insulin analogues exhibiting increased mitogenic potency. Biochem J. 1996;315:271-279 (C) the Biochemical Society 
a range of insulin analogues, the correlation between IGF$1 \mathrm{R}$ binding and mitogenic potency was more evident than the correlation between the insulin receptor dissociation rate and mitogenic potency [19]. However, unfortunately none of the knockdown studies included insulin X10 in the study of cellular growth, and these studies were carried out with cells expressing many IGF-1Rs, which may result in a bias towards IGF-1 mediated effects. Finally, blockade of IGF-1R-mediated growth effects using antibody directed against the receptor, almost completely abolished the effect of IGF-1 whereas the effect of insulin X10 was only partially blocked [21]. Thus, it is still unknown whether increased affinity for the IGF-1R or increased half-life of the receptor-ligand complex plays the more important role for the observed increased mitogenic potency, and consequently also whether either of these characteristics can explain the tumour findings in rats with insulin X10.

Several studies have examined the cellular effects of insulin X10 in more detail in the light of the recent progress in our understanding of insulin and IGF-1 signalling pathways.

The decreased dissociation rate was shown to be accompanied by a sustained signalling and phosphorylation of IR and downstream elements including IRS1, Shc and focal adhesion kinase (FAK) $[24,27]$. This could be mere timing of signalling favouring phosphorylation fingerprinting that was directed towards mitogenic signalling. Moreover, an altered timing of signalling could lead to interactions with and/or phosphorylation of cytosolic signalling molecules that are otherwise not encountered. In this respect it is noteworthy that the natural secretion pattern of IGF-1 is more continuous than that of insulin. Cellular localisation of the signal could also play a role, since it has been shown that the cellular processing of insulin X10 is clearly different from that of human insulin $[12,28]$. Thus, stimulation with insulin X10 could result in increased phosphorylation after internalisation of the receptorligand complex and consequently induce phosphorylation in other subcellular compartments.

In an extensive study of ligand-induced phosphorylation of many different intracellular proteins it was noted that the phosphorylation pattern seen after stimulation of Michigan Cancer Foundation-7 (MCF-7) cells with insulin X10 was different from that after stimulation with insulin. An asymmetrical preferential phosphatidylinositol 3-kinase (PI3K) pathway activation and, specifically, stimulation of protein translation, was seen after stimulation with insulin X10 [29]. Other studies have also found an important role of the PI3K pathway [25], whereas yet more results seem to favour a more pronounced stimulation of the extracellular signal-regulated kinase (ERK) pathway [30]. Thus, while it is apparent that insulin X10 and other ligands can induce a signalling pattern different from that of insulin, the exact pattern is not entirely obvious and, clearly, more studies comparing the relative activation of the different pathways are needed. In this respect it is imperative to stress the importance of performing full dose-response curves, since investigation of only one or few doses may result in erroneous conclusions [31]. In such dose-response experiments, preliminary results indicate that insulin X10 might induce a preferential phosphorylation of the more N-terminal (Y972 and Y1158) phosphorylation sites on IR itself compared with the most C-terminal phosphorylation site (Y1334) [32]. Therefore phosphorylation of the kinase domain seemed to correlate with the mitogenic potency of insulin X10, while phosphorylation of the C-terminal correlated with the metabolic potency of X10. These findings suggest that kinase domain phosphorylation might be a major determinant of mitogenic potency, but this remains to be confirmed in experiments with a panel of mitogenic insulin analogues and in setups using different cell types and stimulation periods.

Preferential signalling could arise from both of the molecular characteristics shown in Fig. 2, but in addition the issue of different IR isoforms and IR/IGF-1R hybrids must be taken into account. It has been speculated that an increase in the mitogenic properties of an insulin analogue could alternatively (or additionally) reflect a binding preference for the shorter IR-A isoform of IR relative to the longer IR-B isoform [30,33]. This hypothesis is derived from the observation that IR-A has high affinity for binding IGF-2 and is extensively expressed in fetal tissue, where it mediates growth responses. IR-A expression is also associated with undifferentiated cells, and overexpression occurs in some cancer cells. This raises the possibility that this IR isoform may be relevant for the mitogenesis of cancer cells [33]. However, studies have shown that both the binding affinity and the activation of the two IR isoforms are very similar after stimulation with insulin $\mathrm{X} 10[22,30,34,35]$. Cells with both IR and IGF-1R will also express heterodimer hybrid receptors [36, 37]. The physiological significance of these receptors is probably low. Hybrid receptors have been shown to preferentially bind IGF-1, whereas insulin is bound with a much lower affinity regardless of which IR isoform is part of the heterodimer, and several reports have shown that insulin fails to activate hybrid receptors [37]. The binding of insulin X10 to hybrid receptors is unknown at present, but is currently under investigation. Some studies have found an increase in the maximal attainable response after stimulation with insulin $\mathrm{X} 10[18,21]$, whereas this has not been found by others $[11,19,38]$. However, it remains a possibility that in some cell types and for some responses, insulin X10 may be able to induce a higher response than human insulin. This could be explained by the presence of IGF-1R and/or hybrids. 


\section{Future perspectives}

Despite the major advances in our understanding of insulin action and signalling the relative roles of IR and IGF-1R in contributing to the increased mitogenic effects of insulin $\mathrm{X} 10$ are still not fully understood and investigations in this area are still ongoing. What is clear is that interactions with both IGF-1R and IR, as well as downstream signalling and biological effects, need to be assessed if the safety of new insulin analogues is to be assured. As in vitro results go only so far in explaining in vivo effects, it would be desirable to define a better tumour assessment model so that we can more effectively predict whether an insulin analogue is likely to promote tumour growth. Although the in vivo rat model that revealed the tumour-promoting effects of insulin X10 is still used today, it is not ideal for long-acting insulins. The relatively high doses of rapidacting insulin X10 at which tumours became apparent in this model cannot be used with long-acting insulins because the rats would die as a result of hypoglycaemia. Therefore, alternative models such as those in which tumour formation is accelerated by stimulation with various chemicals (e.g. DMBA), or models in which tumours are transplanted into diabetic animals, are currently being investigated.

As for insulin X10, its promise of being an effective new rapid-acting insulin analogue turned out to be short lived. Despite this, it has proven to be an important link in the current issue of the molecular safety of insulin analogues, and insulin X10 is now recommended by the European Agency for the Evaluation of Medicinal Products as the positive control in insulin analogue mitogenicity studies.

Acknowledgements The authors acknowledge the editorial assistance of M. Pogson and R. Holmes of Watermeadow Medical, Witney, $\mathrm{UK}$, in the preparation of this article, which has been supported by Novo Nordisk A/S, Bagsvaerd, Denmark.

Duality of interest B. Falck Hansen, P. Kurtzhals and A Dejgaard are employees and shareholders, Novo Nordisk. A. B. Jensen is a previous employee and current shareholder, Novo Nordisk. D. Russell-Jones has received research funding or Advisory board or lecture fee Honoraria from Eli Lilly, Novo Nordisk, GlaxoSmithKline, Aventis, Takeda, Novartis and Pfizer.

\section{References}

1. Coughlin SS, Calle EE, Teras LR, Petrelli J, Thun MJ (2004) Diabetes mellitus as a predictor of cancer mortality in a large cohort of US adults. Am J Epidemiol 159:1160-1167

2. Ogunleye AA, Ogston SA, Morris AD, Evans JM (2009) A cohort study of the risk of cancer associated with type 2 diabetes. Br J Cancer 101:1199-1201

3. Renehan AG, Tyson M, Egger M, Heller RF, Zwahlen M (2008) Body-mass index and incidence of cancer: a systematic review and meta-analysis of prospective observational studies. Lancet 371:569-578
4. Tran TT, Medline A, Bruce WR (1996) Insulin promotion of colon tumors in rats. Cancer Epidemiol Biomark Prev 5:1013-1015

5. Bodmer M, Meier C, Krähenbühl S, Jick SS, Meier CR (2010) Long-term metformin use is associated with decreased risk of breast cancer. Diabetes Care 33:1304-1038

6. Currie CJ, Poole CD, Gale EA (2009) The influence of glucoselowering therapies on cancer risk in type 2 diabetes. Diabetologia 52:1766-1777

7. Colhoun HM, SDRN Epidemiology Group (2009) Use of insulin glargine and cancer incidence in Scotland: a study from the Scottish Diabetes Research Network Epidemiology Group. Diabetologia 52:1755-1765, erratum 52:2469

8. Jonasson JM, Ljung R, Talbäck M, Haglund B, Gudbjörnsdòttir S, Steineck G (2009) Insulin glargine use and short-term incidence of malignancies - a population-based follow-up study in Sweden. Diabetologia 52:1745-1754

9. Hemkens LG, Grouven U, Bender R et al (2009) Risk of malignancies in patients with diabetes treated with human insulin or insulin analogues: a cohort study. Diabetologia 52:1732-1744

10. Brange J, Ribel U, Hansen JF et al (1988) Monomeric insulins obtained by protein engineering and their medical implications. Nature 333:679-682

11. Schwartz GP, Burke GT, Katsoyannis PG (1987) A superactive insulin: [B10-aspartic acid]insulin(human). Proc Natl Acad Sci USA 84:6408-6411

12. Drejer K (1992) The bioactivity of insulin analogues from in vitro receptor binding to in vivo glucose uptake. Diab Metab Rev $8: 259-285$

13. Blundell T, Dodson G, Hodgkin D, Mercola D (1972) Insulin: the structure in the crystal and its reflection in chemistry and biology. Adv Protein Chem 26:279-402

14. Kang S, Brange J, Burch A, Vølund A, Owens DR (1991) Subcutaneous insulin absorption explained by insulin's physicochemical properties. Evidence from absorption studies of soluble human insulin and insulin analogues in humans. Diabetes Care 14:942-948

15. Kang S, Creagh FM, Peters JR, Brange J, Vølund A, Owens DR (1991) Comparison of subcutaneous soluble human insulin and insulin analogues (AspB9, GluB27; AspB10; AspB28) on mealrelated plasma glucose excursions in type I diabetic subjects. Diabetes Care 14:571-577

16. Nielsen FS, Jørgensen LN, Ipsen M, Voldsgaard AI, Parving HH (1995) Long-term comparison of human insulin analogue B10Asp and soluble human insulin in IDDM patients on a basal/bolus insulin regimen. Diabetologia 38:592-598

17. Drejer K, Kruse V, Larsen UD, Hougaard P, Bjørn S, Gammeltoft S (1991) Receptor binding and tyrosine kinase activation by insulin analogues with extreme affinities studied in human hepatoma HepG2 cells. Diabetes 40:1488-1495

18. Bornfeldt KE, Gidlöf RA, Wasteson A, Lake M, Skottner A, Arnqvist HJ (1991) Binding and biological effects of insulin, insulin analogues and insulin-like growth factors in rat aortic smooth muscle cells. Comparison of maximal growth promoting activities. Diabetologia 34:307-313

19. Kurtzhals P, Schäffer L, Sørensen A et al (2000) Correlations of receptor binding and metabolic and mitogenic potencies of insulin analogs designed for clinical use. Diabetes 49:999-1005

20. Slieker LJ, Brooke GS, DiMarchi RD et al (1997) Modifications in the B10 and B26-30 regions of the B chain of human insulin alter affinity for the human IGF-I receptor more than for the insulin receptor. Diabetologia 40(Suppl 2):S54-S61

21. Milazzo G, Sciacca L, Papa V, Goldfine ID, Vigneri R (1997) ASPB10 insulin induction of increased mitogenic responses and phenotypic changes in human breast epithelial cells: evidence for enhanced interactions with the insulin-like growth factor-I receptor. Mol Carcinog 18:19-25 
22. Sommerfeld MR, Müller G, Tschank G et al (2010) In vitro metabolic and mitogenic signaling of insulin glargine and its metabolites. PLoS ONE 5:e9540

23. Kohn WD, Micanovic R, Myers SL et al (2007) pI-shifted insulin analogs with extended in vivo time action and favorable receptor selectivity. Peptides 28:935-948

24. Hansen BF, Danielsen GM, Drejer K et al (1996) Sustained signalling from the insulin receptor after stimulation with insulin analogues exhibiting increased mitogenic potency. Biochem $\mathrm{J}$ 315:271-279

25. Eckardt K, May C, Koenen M, Eckel J (2007) IGF-1 receptor signalling determines the mitogenic potency of insulin analogues in human smooth muscle cells and fibroblasts. Diabetologia 50:2534-2543

26. Shukla A, Grisouard J, Ehemann V, Hermani A, Enzmann H, Mayer D (2009) Analysis of signaling pathways related to cell proliferation stimulated by insulin analogs in human mammary epithelial cell lines. Endocr Relat Cancer 16:429-441

27. Berti L, Kellerer M, Capp E, Häring HU (1997) Leptin stimulates glucose transport and glycogen synthesis in $\mathrm{C} 2 \mathrm{C} 12$ myotubes: evidence for a P13-kinase mediated effect. Diabetologia 40:606609

28. Hamel FG, Siford GL, Fawcett J, Chance RE, Frank BH, Duckworth WC (1999) Differences in the cellular processing of AspB10 human insulin compared with human insulin and LysB28ProB29 human insulin. Metabolism 48:611-617

29. Oleksiewicz MB, Bonnesen C, Hegelund AC et al. (2010) Comparison of intracellular signalling by insulin and the hypermitogenic AspB10 analogue in MCF-7 breast adenocarcinoma cells. J Appl Toxicol. doi:10.1002/jat.1590

30. Sciacca L, Cassarino MF, Genua M et al (2010) Insulin analogues differently activate insulin receptor isoforms and post-receptor signalling. Diabetologia 53:1743-1753
31. Pollak M, Russell-Jones D (2010) Insulin analogues and cancer risk: cause for concern or cause célèbre? Int J Clin Pract 64:628 636

32. Hansen BF, Pia J (2010) Detailed analysis of insulin receptor phosphorylation after stimulation with insulin X10. Ir10: XIth International Symposium on Insulin receptors and Insulin Action (Abstract no. P09.95). http://www.irm10.com/Final-AbstractBook.pdf. Accessed 16 March 2011

33. Belfiore A, Frasca F, Pandini G, Sciacca L, Vigneri R (2009) Insulin receptor isoforms and insulin receptor/insulin-like growth factor receptor hybrids in physiology and disease. Endocr Rev 30:586-623

34. Glendorf T, Knudsen L, Stidsen CE et al. (2010) Evaluation of the metabolic to mitogenic potency ratio for B10-substituted insulin analogs. Ir10: XI International Symposium on Insulin Receptors and Insulin Action. Naples, Italy, 2010. Poster P09.94. www. irm10.com/Final-Abstract-Book.pdf. Accessed 16 May 2011

35. Hansen BF, Stidsen CE, Glendorf T, Lundby A, Hegelund AC, Bonnesen C (2010) Safety analysis of basal insulins: mitogenic potency and receptor binding. Diabetologia 53(suppl 1):S22

36. Pandini G, Frasca F, Mineo R, Sciacca L, Vigneri R, Belfiore A (2002) Insulin/insulin-like growth factor I hybrid receptors have different biological characteristics depending on the insulin receptor isoform involved. J Biol Chem 277:39684-39695

37. Slaaby R, Schäffer L, Lautrup-Larsen I et al (2006) Hybrid receptors formed by insulin receptor (IR) and insulin-like growth factor I receptor (IGF-IR) have low insulin and high IGF-1 affinity irrespective of the IR splice variant. J Biol Chem 281:2586925874

38. Bonnesen C, Nelander GM, Hansen BF et al (2010) Synchronization in $\mathrm{G} 0 / \mathrm{G} 1$ enhances the mitogenic response of cells overexpressing the human insulin receptor $\mathrm{A}$ isoform to insulin. Cell Biol Toxicol 26:293-307 